\title{
Frequency Noise of a Normal Dispersion Microresonator-based Frequency Comb
}

\author{
Attila Fülöp, ${ }^{1}$ Mikael Mazur, ${ }^{1}$ Abel Lorences-Riesgo, ${ }^{1}$ Pei-Hsun Wang, ${ }^{2}$ Yi Xuan,,${ }^{2,3}$ Dan E. Leaird, ${ }^{2}$ \\ Minghao Qi, ${ }^{2,3}$ Peter A. Andrekson, ${ }^{1}$ Andrew M. Weiner, ${ }^{2,3}$ and Victor Torres-Company ${ }^{1}$ \\ ${ }^{1}$ Photonics Laboratory, Department of Microtechnology and Nanoscience, Chalmers University of Technology, SE-41296 Göteborg, Sweden. \\ ${ }^{2}$ School of Electrical and Computer Engineering and ${ }^{3}$ Birck Nanotechnology Center, Purdue University, West Lafayette, IN 47907-2035, USA. \\ Authore-mail address: attila.fulop@ chalmers.se
}

\begin{abstract}
Using delayed self-heterodyne coherent detection, we characterized the FM noise across the C-band of a widely spaced microresonator-based frequency comb. The resulting linewidth depends on both the pump laser and the comb line position. OCIS codes: (060.1660) Coherent communications; (190.4390) Nonlinear optics, integrated optics.
\end{abstract}

\section{Introduction}

Recent experiments have demonstrated that microresonator-based Kerr combs are promising multi-wavelength integrated light sources for use in wavelength-division multiplexed (WDM) coherent communication systems, both in high-capacity single-span [1] and long-haul systems [2]. Kerr combs implemented in silicon nitride high-Q microresonators [3] offer a viable path to co-integrate the technology platform with additional transmitter components [4].

In order to use higher-order modulation formats with the lines of the Kerr comb, a particular emphasis must be placed on their linewidth properties. In microresonator combs this will be ultimately dictated by the laser pump linewidth and the nonlinear comb dynamics [5]. Unlike laser arrays, chip-scale comb generators may show a distinct linewidth behavior for different frequency lines. However, this topic has not been thoroughly investigated for Kerr combs.

Here, we carry out an experimental study of the linewidth variation across a low-noise Kerr comb centered in the C-band. The comb operated in the coherent modulation instability regime [6]. We analyze the FM noise of the instantaneous frequency [7], which allows us to distinguish the dominant frequency noise contributions for different time scales. We find that in the region relevant for coherent optical communications, the linewidth increases slightly as it moves away from the pump, regardless of the laser pump source. Although the impact is not severe for $20 \mathrm{Gbaud}$ 16QAM modulation when using a high-quality laser pump, these results point out phase noise dynamics that may be relevant for higher-order constellations.

\section{Motivation - frequency noise impact on coherent communications}

In the following we describe an experiment that motivated us to look further into the linewidth properties of the comb. We used a Kerr comb implemented in a high-Q $\left(\sim 10^{6}\right)$ microresonator with $230 \mathrm{GHz}$ free spectral range (FSR). The fundamental mode displays normal dispersion and, owing to linear coupling to a higher-order mode, the comb is switched on via modulation instability [8]. The comb is coherent and stable over long term in a temperature-controlled environment. Figure 1a) shows a schematic for the Kerr comb generation while Fig. 1b) shows a typical spectrum, before spectral flattening, that covers the C-band with the lines spaced every 3 FSRs $(690 \mathrm{GHz})$. The comb is in a low-noise state, as indicated by the electrical spectrum analyzer [6], see Fig. 1c). Next, to emulate a transmission scenario, we equalized the Kerr comb lines in power using a pulse shaper and an EDFA [2]. The comb lines were modulated with 20 Gbaud polarization multiplexed 16QAM data using an arbitrary waveform generator (AWG) and a polarization multiplexing stage, see Fig. 1d). We assessed the bit-error rate (BER) vs. optical signal-to-noise ratio (OSNR) at $0.1 \mathrm{~nm}$ bandwidth in back-to-back for every line using an additional noise-loading stage. The receiver consisted of a tunable local oscillator laser, a coherent receiver and a $23 \mathrm{GHz}$ real-time oscilloscope. The BER was calculated after processing the received data by offline DSP containing a decision-directed least mean square equalizer and phase tracker.

Figure 1e) shows that a different OSNR is required depending on the line in order to achieve the same BER of $10^{-3}$. Although the penalty difference is less than $1 \mathrm{~dB}$, it seems to follow roughly the same wavelength variation as the equivalent linewidth between the measured comb line and the local oscillator. The equivalent linewidth was calculated digitally from the DSP data in the $2-20 \mathrm{MHz}$ region of the FM noise using the method described in the following section. In order to assess whether the linewidth variation is a feature inherent to the comb we realized a careful measurement of the FM noise spectrum of each line using self-delayed heterodyne coherent detection [9]. 
a)
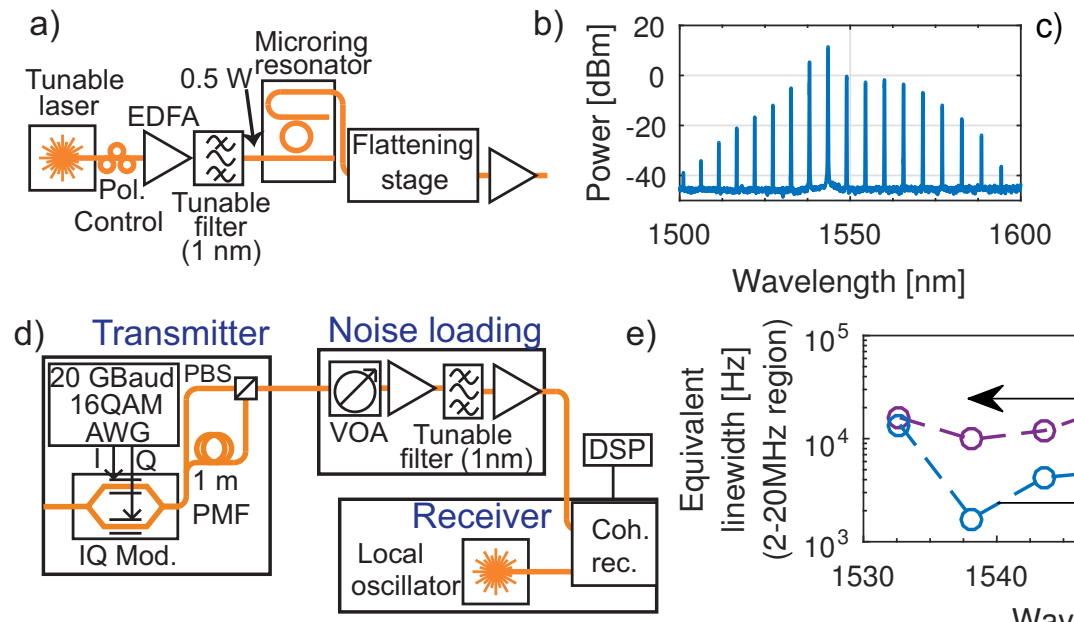
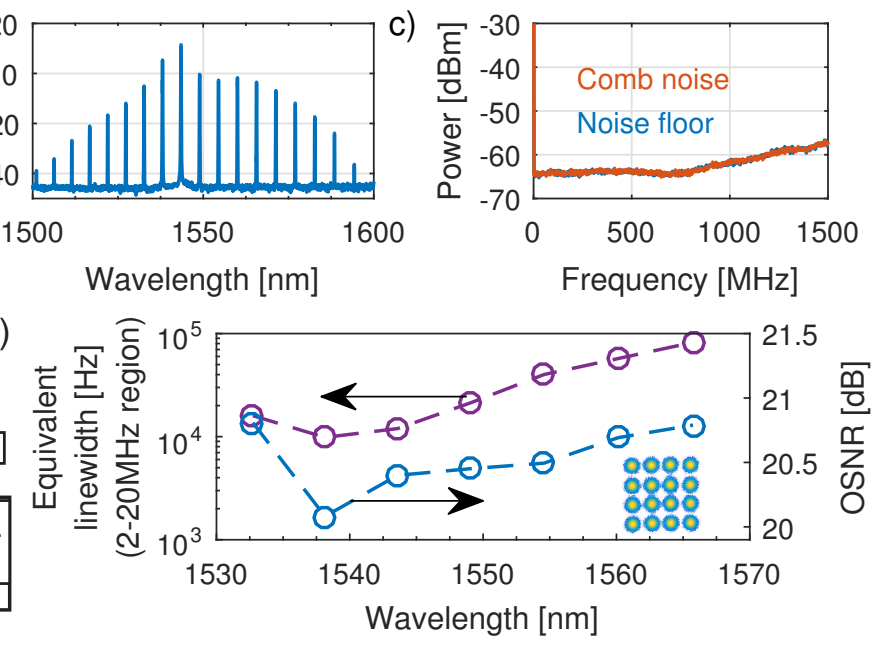

Fig. 1. a) Setup for generating and flattening the frequency comb. b) Optical and c) electrical power spectrum of the comb running in a mode with low amplitude noise. d) Noise loading setup for measuring implementation penalty. e) The equivalent combined linewidth of the comb and a free-running LO shown together with the corresponding OSNR requirement for a $16 \mathrm{QAM}$ system operated at a receiver $\mathrm{BER}=10^{-3}$. The theoretical OSNR limit at $18.6 \mathrm{~dB}$ means that the penalty is around $2 \mathrm{~dB}$. The inset shows a constellation diagram for the comb line at $1538 \mathrm{~nm}$ at $\mathrm{BER}=10^{-3}$.

\section{Line-resolved linewidth measurements}

The phase of an optical light wave can not be measured directly. By using an interferometric setup, such as the one shown in Fig. 2a), one can however measure its drift over time. In the measurement setup, after filtering out one comb line and attenuating it to a fixed level, we split the light in two arms that we then fed into a coherent receiver. One of the arms was delayed by a time longer than the coherence time of the pump laser using a fiber. It was also shifted by $27 \mathrm{MHz}$ using an acousto-optic modulator to avoid artifacts from the DC block in the coherent receiver. The outputs of the coherent receiver were connected to two ports of a $6.25 \mathrm{GS} / \mathrm{s}$ real-time oscilloscope from which we could extract the signal phase after compensating for the IQ imbalance in the receiver and the linear phase shift caused by the acousto-optic modulator. To extract useful information about the phase noise, we then calculate the FM noise spectrum, i.e. the power spectral density of the instantaneous frequency, $S_{\mathrm{FM}}[7]$ :

$$
S_{\mathrm{FM}}(v)=\left\langle|\mathfrak{F}[f(t)]|^{2}\right\rangle=\left\langle\left|\mathfrak{F}\left[\frac{1}{2 \pi} \frac{\partial \phi(t)}{\partial t}\right]\right|^{2}\right\rangle .
$$

In Eq. $1, \mathfrak{F}$ denotes the Fourier transform while \langle\rangle denotes ensemble averaging. In the case of a laser line with Lorentzian shape, $S_{\mathrm{FM}}$ is flat and one can directly extract the combined linewidth by multiplying it with $\pi$ [7]. In the context of optical communications, the low frequency components of the phase noise can typically be tracked and compensated for successfully in DSP. The noise floor, or the Lorentzian-like linewidth therefore has a more significant effect on the final BER [9].

Both the reference pump laser as well as the comb lines were then measured using the setup in Fig. 2a). The FM noise spectrum of the tunable external cavity laser used to pump the microresonator can be seen in Fig. 2b) together with one of the comb lines. The pump laser noise floor is at $5000 \mathrm{~Hz}^{2} / \mathrm{Hz}$, translating to a Lorentzian-like combined linewidth of approximately $16 \mathrm{kHz}$ in close agreement to the specifications. Since the phase noise of the comb lines did not differ significantly from each other, an alternative comparison method was needed. For this purpose two spectral regions were selected and averaged over: $0.1-1 \mathrm{MHz}$ and $2-20 \mathrm{MHz}$, shown as shaded areas in Fig. 2b).

To ensure that the setup was wavelength-independent, the reference pump laser was also tuned and measured at all wavelengths matching the lines of the frequency comb. Additionally, to assess the effect of the pump laser, the microresonator was also pumped with a different, higher linewidth, external cavity laser. The results can be seen in Fig. 2c) where the FM noise spectrum values at the chosen frequencies have been multiplied by $\pi$ to get a Lorentzian equivalent linewidth. The tuned reference laser is shown as black circles. The comb lines generated using the same laser are marked with blue circles while the comb lines generated by the high linewidth laser are marked in red. 
a)

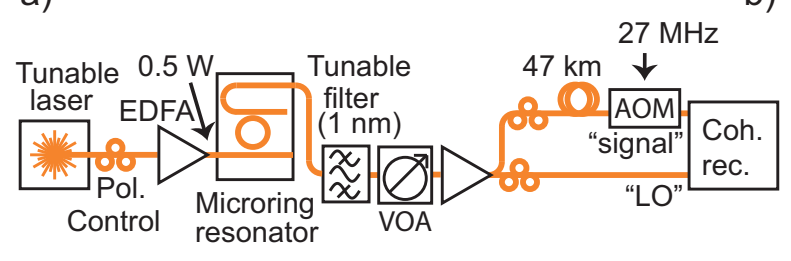

c)

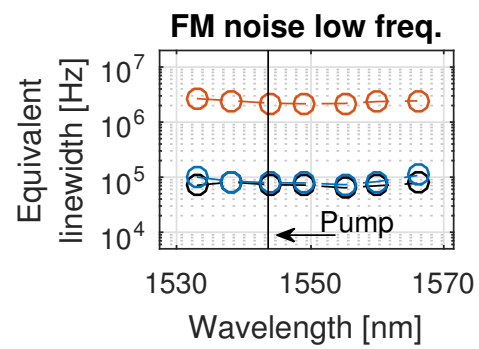

b)

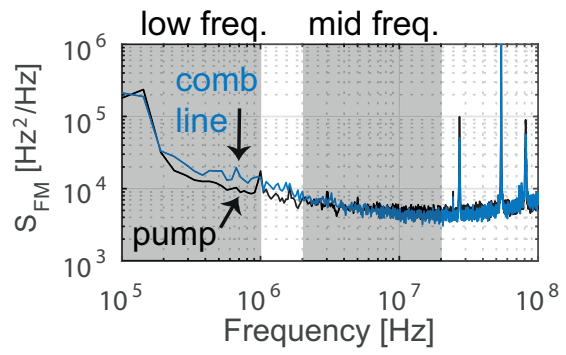

Fig. 2. a) Schematic of the phase noise measurement setup. b) The FM noise spectrum of the pump laser and one of the comb lines. The peaks at 27,54 , and $81 \mathrm{MHz}$ are harmonics present due to the AOM. The regions marked in red between $0.1-1 \mathrm{MHz}$ and $2-20 \mathrm{MHz}$ were each averaged and used for the later comparisons. c) The FM noise components at the low and the mid frequency of the reference pump (black), the comb (blue), and the comb pumped with a higher linewidth laser (red). In both cases, the comb was pumped at the same resonance, at $1543.5 \mathrm{~nm}$. The rightmost plot shows a zoomed-in view of the mid frequency noise level.

\section{Conclusions}

We have characterized the FM noise of a normal dispersion Kerr comb providing information about the comb linewidth in a frequency-resolved manner. In the low-frequency region (corresponding to self-heterodyne ESA-based beatnote linewidth measurements), our results indicate that the linewidth of the comb lines is largely dictated by the pump laser and does not vary significantly from line to line. However, the instantaneous linewidth in the short temporal scale, that is relevant for phase tracking in coherent communication systems, displays an asymmetric behavior with respect to wavelength. The linewidth can vary by more than a factor of ten, depending on the pump laser. While different types of Kerr combs might have different scaling effects, further investigations need to be done for a complete physical understanding. These results extend previous observations of equal linewidth across the bandwidth of Kerr combs and call for extra caution and laser pump requirements when using higher-order constellations.

\section{References}

[1] J. Pfeifle et al., "Full C and L-band transmission at 20 Tbit/s using cavity-soliton Kerr ...," CLEO Postdeadline Papers, JTh5C.8 (2015).

[2] A. Fülöp et al., "Long-haul coherent transmission using a silicon nitride microresonator-based frequency comb as ...," CLEO, SM4F.2 (2016).

[3] J. S. Levy et al., "CMOS-compatible multiple-wavelength oscillator for on-chip optical interconnects," Nat. Photon. 4, 37 (2010).

[4] W. D. Sacher et al., "Multilayer silicon nitride-on-silicon integrated photonic platform for 3D ...," CLEO Postdeadline Papers, JTH4C.3 (2016).

[5] P. Liao et al., "Dependence of Kerr comb linewidth and coherent system performance on the pump linewidth," ECOC, Th.2.P2.SC1.5 (2016).

[6] P.-H. Wang et al., "Observation of correlation between route to formation, coherence, and communication ...," Opt. Express 20, 29284 (2012).

[7] K. Kikuchi, "Characterization of semiconductor-laser phase noise and estimation of bit-error rate ...," Opt. Express 20, 5291 (2012).

[8] Y. Liu et al., "Investigation of mode coupling in normal-dispersion silicon nitride microresonators for Kerr frequency ...," Optica 1, 137 (2014).

[9] T. N. Huynh et al., "Phase noise characterization of SGDBR lasers using phase modulation ...," J. Lightwave Technol. 31, 1300 (2013).

We acknowledge support from the European Research Council (ERC-2011-AdG-291618 PSOPA), the Swedish Research Council (VR) and the KA Wallenberg foundation. The effort at Purdue was supported in part under grants from NSF (ECCS-150957), DARPA (W31P40-13-1-001.8) and AFOSR (FA9550-15-1-0211). 